\title{
EL ESTUDIO DE LOS DISCURSOS ALIMENTARIOS DESDE UNA PERSPECTIVA CRÍTICA
}

\section{THE STUDY OF FOOD DISCOURSES FROM A CRITICAL PERSPECTIVE}

\author{
SUSANA RodRÍGUEZ BARCIA* \\ Universidade de Vigo \\ susanarbarcia@gmail.com \\ http://orcid.org/0000-0001-7035-6019
}

Recibido: 09/07/2019

Aceptado: 06/02/2020

\begin{abstract}
Resumen
La alimentación, por su condición esencial como parte indisociable de la naturaleza humana, constituye una de las temáticas más relevantes para la población mundial y, por ello, se conforma como un objeto de estudio fundamental desde los ámbitos económico, antropológico, médico y, por supuesto, discursivo. Este último interesa especialmente en cuanto que las sociedades contemporáneas se caracterizan por la información excedente y normalizada sobre la alimentación que se vehicula a través de las publicaciones científicas y divulgativas.

Este contexto propicia el interés que, fundamentealmente desde el año 2000, despierta el estudio de los discursos alimentarios dentro del ámbito del análisis del discurso con perspectiva crítica, pues los discursos de la alimentación resultan determinantes en la conformación del
\end{abstract}

\begin{abstract}
Because eating is such an integral part of human activity and survival, food is one of the most important topics for the world population and, therefore, is an essential subject of study in the fields of economics, anthropology, medicine and, of course, discourse. The latter field is of particular interest because contemporary societies are affected by the excessive amount of information on food that is transmitted through scientific and informational publications.

This context propitiates the interest that, fundamentally since the year 2000, awakens the study of food discourses within the scope of discourse analysis with a critical perspective, because the discourses of food are determinants in the conformation of the ethos of the new figure of consumers.
\end{abstract}

\footnotetext{
* La investigación sobre los discursos alimentarios desde una perspectiva crítica se está desarrollando con la colaboración del grupo de investigación GRADES, de la Universidade de Vigo.
}

Para citar este artículo / To cite this article: Rodríguez Barcia, Susana (2020). El estudio de los discursos alimentarios desde una perspectiva crítica. ELUA, 34: 175-191. https://doi.org/10.14198/ ELUA2020.34.8

Enlace / Link: https://doi.org/10.14198/ELUA2020.34.8 
ethos de la nueva figura de consumidores y consumidoras.

En este artículo se resumen las principales investigaciones que vinculan ideología y discurso alimentario realizadas en clave principalmente lingüística, más allá de los estudios de comunicación y marketing. Se conforma así como un estado de la cuestión y una reflexión teórica de utilidad para afrontar el abordaje de investigaciones lingüísticas y críticas de los discursos alimentarios.

PALABRAS CLAVE: comunicación científica, discurso alimentario, discurso científico, ideología, identidad
This paper summarizes the main research that links ideology and food discourse made mainly linguistic key, beyond the studies of communication and marketing. It is thus formed as a state of the question of utility to face the approach of linguistic and critical investigations of the alimentary discourses.

KEYWORDS: Scientific discourse, publications on food, scientific discourse, ideology, identity

\section{INTRODUCCIÓN}

La alimentación es la columna vertebral de la humanidad. Su papel en el sustento, organización y desarrollo de las distintas comunidades humanas resulta indiscutible. Es por ello que los discursos sobre las prácticas alimentarias suponen un objeto de estudio fundamental en el ámbito de la investigación humanística. En general, todos los discursos de carácter científico son percibidos por la sociedad como suficientemente legitimados como para justificar modificaciones en los hábitos de vida y consumo y, cuando se trata de aspectos relativos a la nutrición y a la salud, la receptividad popular es todavía mayor siempre que se entienda que conocer e interiorizar estos discursos va a permitir tener mejor calidad de vida. Los discursos científicos que tienen como tema principal la alimentación en cualquiera de sus dimensiones (social, nutricional, ética, ecológica, cultural, etc.) tienen, pues, la capacidad de influir directamente sobre la sociedad, bien en aspectos ideológicos sin implicación práctica, bien en dinámicas concretas de consumo. Pero la cuestión de fondo es que a los discursos científicos y de divulgación científica se les ha atribuido un carácter simbólico que hace que la producción de textos especializados ya no sea solo un vehículo para la expresión de la ciencia, sino una plataforma que contribuye a la promoción de diferentes estilos de vida y a la demonización de otros. Si bien es cierto que la cultura dominante impone una forma particular de ciencia, también es relevante que en toda comunicación científica se establece además una relación inicua entre emisión y recepción o, más concretamente, un desequilibrio de conocimientos entre las personas que escriben la ciencia y las personas legas o semilegas que la reciben (Gouveia y Alexandre 2009).

Aunque la "ciencia" hace referencia a un conjunto de conocimientos que se recaban a partir de la investigación y de la reflexión que ofrecen la posibilidad de generalizar principios y leyes fundamentales, la realidad es que "lo científico" parece haberse sometido a un proceso imparable de vulgarización (Jacobi 1987), en el sentido de que se está reduciendo cada vez más a un conjunto de conocimientos superficiales que se manejan por el conjunto de la sociedad sin profundizar en su veracidad o en la base de sus lógicas subyacentes. Esto supone que en la descodificación de los textos especializados se presente una condición de desventaja por parte de las personas receptoras, que asumirán en gran medida la verdad de 
los contenidos como una condición intrínseca al conocimiento científico, y que subjetivizarán esa realidad asumiéndola como propia. A este hecho ha de añadirse un factor no menos importante que ha convertido en central el tema de la alimentación, la polarización que están sufriendo en las últimas décadas tanto el debate sobre la seguridad alimentaria (Muñoz 2002), como la reflexión sobre la idoneidad y carácter ético de ciertas prácticas alimentarias como el vegetarianismo, veganismo o la slow food, por ejemplo (Brunori, Malandrin \& Rossi 2013); o el debate sobre la producción ecológica y la implementación de nuevas tecnologías en los procesos de cultivo (Cook 2004). Esta diversificación de opiniones contrapuestas evidencia, a partir del desacuerdo científico que ponen de manifiesto, la toma de postura de las investigaciones, su sesgo, así como sus condicionamientos ideológicos y materiales. Por lo tanto, pese a la concepción popular, la comunicación de la ciencia no es en absoluto objetiva ni neutral, sino que está controlada por los mecanismos de poder y se mueve por intereses de diversa índole o, sencillamente, se ve condicionada por factores culturales aprehendidos, es decir, concebidos sin someter a un juicio crítico.

Con todo, a pesar de la conciencia cada vez mayor de que el discurso científico es discrepante, falible, tendencioso y adolece de las debilidades propias de cualquier producción intelectual, el valor simbólico otorgado a las textualidades científicas sigue disponiéndolas en un lugar de privilegio, como discursos que profesan la verdad y en los que no suele tener cabida la subjetividad. Esta tensión entre la objetividad pretendida y la subjetividad inherente a los textos hace necesario profundizar en el estudio de la presencia de ideología en los discursos científicos y divulgativos de la alimentación como manifestaciones que, por su incidencia social en las prácticas de consumo y en los hábitos de vida (Cook 2010), constituyen un objeto de estudio fundamental para la lingüística. Es por ello que en este artículo se recogen algunas de las principales investigaciones que se vienen desarrollando en relación con el estudio lingüístico-ideológico de los discursos alimentarios, con el fin de establecer un panorama general y de facilitar de este modo los futuros acercamientos a un ámbito de estudio relativamente reciente.

\section{LAS INVESTIGACIONES GENERALES SOBRE LOS DISCURSOS QUE ABOR- DAN CUESTIONES RELATIVAS A LA ALIMENTACIÓN}

Las investigaciones que abordan el estudio de la seguridad y prácticas alimentarias desde una perspectiva discursiva tienen a partir del año 2000, y hasta la actualidad, su momento de mayor difusión e impacto social. Aunque desde los años ochenta del siglo XX ya se venían desarrollando diversas investigaciones sobre el discurso de la alimentación, es con el siglo XXI cuando esta temática cobra una verdadera relevancia en los estudios realizados desde la lingüística (Cook 2004), así como desde otros ámbitos como el de la comunicación, la antropología y la sociología. Oseguera Parra (2001) ya concluía que en la investigación sobre la temática alimentaria confluían distintas disciplinas que habían observado unilateralmente el objeto de estudio según su campo de interés y destaca en sus conclusiones la necesidad de hacer análisis interdisciplinares que permitan una visión de conjunto sobre aspectos en debate actualmente como, por ejemplo, la homogeneización del gusto en el mundo globalizado o la cultura de la alimentación ecológica (Benito Morán 2016). En este sentido, lo cierto es que la investigación lingüística sobre la comida y la alimentación puede ofrecer grandes aportaciones en conjunción con perspectivas de carácter sociológico (Szatrowski 
2014) y también con la visión que proporcionan los estudios de marketing (Mazzacano \& Falzon 2015) o de publicidad y comunicación (Díaz Rojo y Morant 2007).

Por lo tanto, si el estudio de la alimentación desde siempre ha implicado a distintas disciplinas, también el estudio discursivo de la alimentación ha despertado el interés desde diversos ámbitos y ha dado como frutos multitud de investigaciones, casi todas ellas realizadas fuera del ámbito español. Los discursos que han constituido la base de estas investigaciones, su corpus de trabajo, comprenden una tipología muy variada entre la que es posible encontrar artículos de investigación y divulgación, tesis doctorales especializadas (Schaefer 2012), informes institucionales, manuales y libros de texto, monografías científicas y divulgativas, artículos periodísticos (Cassani y Martí 1998; Cook 2004; Demonte 2013; Gómez Oroná 2014; Pérez Martínez 2011), diccionarios (Eberenz 2014), textos publicitarios (Cook, Reed y Twiner 2009), blogs divulgativos y redes sociales (Rousseau 2012; De Choudhury, Sharma y Kiciman 2016; Leigh Burton 2016), entrevistas (Cook, Robbins y Pieri 2004 y 2006; Alvear 2015; Rivarosa y De Longhi 2006), discursos políticos (Cook 2004) e incluso textos fílmicos (Febrer Fernández 2014). Algunas publicaciones recientes abordan el tema a partir de la revisión de un conjunto plural de fuentes documentales que permiten una triangulación de los datos, lo cual proporciona información completa y multidimensional del tema (Cook 2004; Cook, Reed y Twiner 2009; Frye \& Bruner 2012). Las principales temáticas en las que se centran gran parte de las investigaciones tienen que ver con el análisis de las características lingüísticas y las estrategias discursivas de la comunicación científica y de la divulgación en materia de alimentación, los rasgos lingüísticos de la comunicación alimentaria en situaciones de crisis, el estudio de la construcción discursiva de los modelos dominantes de alimentación, la proyección de estereotipos sociales en función de las prácticas alimentarias y, en menor medida, la influencia de los discursos sobre la alimentación en los hábitos de consumo. Aunque de forma muy general, podría decirse que los principales indicadores lingüísticos en los que hay que centrar la investigación crítica del discurso alimentario son los aspectos relativos a la modalización discursiva (especialmente las modalidades epistémica y deóntica); la adjetivación (en especial cuando es axiológica y evaluativa); la elección de temáticas (sobre todo por parte de los medios de comunicación); $\mathrm{y}$, finalmente, el tono (cuando se excede la información para llegar al sensacionalismo o cuando se reviste de científico lo meramente especulativo).

\subsection{De la ciencia a la divulgación científica}

Como marco general a los estudios sobre los discursos de la alimentación se encuentra el análisis previo de la comunicación de la ciencia a partir de los discursos científicos y divulgativos. En estas primeras décadas del siglo XXI es tan grande el auge de los estudios sobre el discurso científico que, en el contexto europeo, ya se han realizado jornadas y encuentros que abordan esta cuestión con carácter monográfico, sobre todo en lo relativo a la construcción discursiva del ethos de autoridad en los textos científicos y divulgativos. Además del análisis de las estrategias lingüísticas y dinámicas de redacción que entran en juego en el proceso de reformulación desde el texto científico al divulgativo, algunos estudios han ido adoptando una perspectiva más crítica y han abordado la cuestión de fondo sobre la presencia de ideologías privativas, generalmente dominantes, en la comunicación científica. 
Desde esta perspectiva analítica, la investigación se ha orientado en distintas direcciones, entre las que destacan los trabajos de carácter general en los que, como se ha referido, se pone en cuestión la objetividad de la comunicación científica y de la divulgación (Luke 2002 o García Negroni 2008); y los que se dirigen a cuestiones más específicas sobre la subjetividad en la comunicación de la ciencia pero a partir de temáticas concretas, como el tratamiento de la producción discursiva sobre el cambio climático, la alimentación ecológica o los alimentos genéticamente modificados, por citar solo algunos de los temas más controvertidos.

Empezando por las aportaciones del primer grupo referido, el paso de la comunicación científica (CC) a la divulgación científica (DC) y las estrategias discursivas que se activan en este proceso han sido ampliamente analizados en todas las tradiciones lingüísticas. Algunos de los estudios clásicos que se suelen destacar en lo relativo a la retórica de la CC son los de Bazerman (1988) y Myers (1991). En el ámbito hispanohablante destacan sin duda los trabajos de Ciapuscio (1993 y 2001), Gallardo (1998), Cassany (2003) o Alcíbar (2007), entre otros. Un trabajo coral, imprescindible para conocer las características del español en los lenguajes de especialidad es el de Alcaraz Varó, Mateo Martínez y Yus Ramos (2007), en el que también se analiza el proceso de trasladar la comunicación científica a divulgación. También existen estudios breves sobre la ideología presente en el discurso en el paso de la ciencia a la DC propia de los materiales didácticos como los libros de texto (Atienza y van Dijk 2010). En esta línea destacan también algunas de las investigaciones desarrolladas en el CELGA-ILTEC (Universidade de Coimbra) dirigidas por el profesor Carlos Gouveia en el marco del análisis crítico de las prácticas discursivas académicas. Trabajos muy recientes como el de Luz et al. (2017) se siguen planteando como pregunta de investigación por el papel que cumple la divulgación científica en la cultura contemporánea, en concreto en lo relativo a temáticas de vida y salud. Este hecho pone de relieve la vitalidad de este objeto de estudio, pues la investigaciones que se centran en los discursos de la ciencia no hacen otra cosa que problematizar una cuestión sobre la que existía cierto grado de silencio aun resultando central en la vida de las sociedades económicamente desarrolladas.

Dos de los principales problemas que se observan en el paso de la comunicación científica de alto nivel de especialización hacia los distintos géneros divulgativos son la teatralización sensacionalista de la ciencia y las consecuencias de la reformulación textual, sobre todo la minimización de incertidumbres. Por un lado, la información añadida en el proceso de traducción desde las lenguas originales de los trabajos (con frecuencia el inglés) hasta las lenguas meta, como ya apunta Alcíbar (2007: 124), implica cierta "teatralización" de la ciencia en la que no es suficiente con la información, por lo que a veces se incurre en la adaptación relativamente libre del texto fuente. Por otro lado, en lo relativo a los procesos de reformulación textual, se ha escrito mucho en cuanto a la expansión, reducción y variación que sufren los textos, pero no tanto sobre la presencia de la subjetividad en los discursos resultantes de la voz de la persona que reformula el texto. Por ejemplo, esta problemática se concreta en la presencia de modalidades deónticas en la comunicación divulgativa de cuestiones alimentarias a través de la presencia de perífrasis verbales (pensemos en casos como "Hay que tener cuidado con frutas como la piña, las uvas, la granada y el plátano, ya que contienen una gran cantidad de azúcar" $A B C, 6 / 7 / 2018$ ); o de modalidades valorativas categóricas a través de adjetivos axiológicos (como en "El error de comer una manzana a media mañana para cuidar la 
dieta", La verdad, 4/7/2018). Estos textos son resultado de procesos de adaptación de hallazgos científicos a medios de consumo masivo, y lo cierto es que este paso puede desencadenar problemas como los detectados, pues se maximizan las certidumbres que en las investigaciones de base aparecen mucho más acotadas y definidas. La lista de ejemplos podría ser muy notable, pero es competencia de la investigación en divulgación científica del discurso alimentario detectar estas claves lingüísticas y establecer, de ser el caso, recomendaciones de redacción para medios de comunicación masivos. Si bien la transición desde el artículo de investigación hacia los géneros divulgativos posee un enorme interés en los estudios discursivos, el paso de la comunicación científica a los manuales didácticos y libros de texto es crucial en los procesos de aprendizaje y, por ello, la presencia de ideología resulta un continuo impedimento para la adquisición de conocimiento desprovista de visiones privativas de los profesionales que ejercen la labor de mediación. Marinkovich (2005), con base en Ciaspucio (2001), identifica algunos de los principales recursos de reformulación textual en los textos de divulgación didáctica, y determina que, en general, se trata de intervenir en el texto para solucionar problemas de comprensión. En este sentido se refiere González Ramírez (2013) al proceso de acercamiento de la ciencia a la educación, que implica que la ciencia se deba ofrecer de una manera accesible para las personas legas en una materia, y que se busque ante todo la comprensión clara de contenidos, lo que conlleva una minimización de incertidumbres que a menudo esconde grandes decisiones de carácter ideológico. Desde el punto de vista lingüístico, esta autora señalaba en este mismo estudio que en los textos de divulgación didáctica predomina la "secuencia explicativa" y que se rompe la "asimetría entre experto y lego" (2013: 21), pero incluso en la explicación se presentan como asumidos y consensuados ciertos principios científicos sobre los que ya no se problematiza. Otros trabajos como el de Muñoz Dagua (2010) someten a análisis el uso de la metáfora léxica en la divulgación científica pero no abordan la presencia de aspectos ideológicos en los textos. También en cuanto al análisis de la metáfora gramatical (Halliday 1985) y su incidencia como vehículo de transmisión ideológica, trabajos recientes (Chamorro, Barletta y Mizuno 2013; Moss 2010; Moss et alii 2013) han centrado su análisis en algunos géneros didácticos para comprobar la relación entre la presencia de ideología que dimana del uso de las metáforas ideacionales (experiencial y lógica) y la socialización y formación en etapas escolares o de aprendizaje. Es sumamente importante este punto, pues no ha sido suficientemente analizada la influencia del discurso privativo de las clases dominantes en los libros de texto y manuales didácticos empleados en las etapas formativas en las que la construcción ideacional del individuo es mucho más frágil, acrítica y susceptible de modificaciones. Es preciso profundizar en la expresión de la causalidad como estrategia lingüística en la comunicación científica relativa a la alimentación y su incidencia en la promoción de determinadas prácticas alimentarias. Por poner solo un ejemplo sencillo, las prácticas omnívoras y carnistas implican una serie de reflexiones y cuestionamientos éticos que en la educación son esquivados con el respaldo de las publicaciones didácticas.

Es evidente que el discurso científico se caracteriza por rasgos comunes a cualquier discurso a los que se incorporan otros elementos que lo singularizan frente al resto, especialmente su capacidad para funcionar como sustento del progreso o como aval de decisiones que condicionan los hábitos de vida, todo ello marcado por su pátina de veracidad y objetividad. Pero, como sintetiza Cassany (2003), la ciencia es un producto cultural, que no 
responde a un orden natural sino a condiciones sociales e históricas; la ciencia se representa con discursos verbales, además de con lenguajes formales, de lo que dimana su dependencia frente a los recursos lingüísticos para su exposición y posterior interpretación. Existe, pues, interdependencia entre la ciencia, los discursos y contextos, y toda esta cadena se inscribe, finalmente, dentro de un mercado social que demanda en cada momento histórico una serie de avances que conllevan una serie de informaciones. Con estas premisas casi toda la investigación actual niega la condición infalible de la comunicación científica, que se ve empañada por las mismas limitaciones de veracidad y objetividad que otros discursos, pues se trata de un producto humano en el que interviene la mediación de diferentes agentes y, por supuesto, del propio lenguaje.

\subsection{Ideología y discurso alimentario}

Si bien es cierto que la cantidad de trabajos que tienen el discurso de la alimentación como objeto de estudio es ingente, lo cierto es que los que se detienen en un análisis del aspecto ideológico constituyen un grupo más reducido, que se estrecha todavía más si se excluyen los que tienen una orientación más sociológica y económica que lingüística. En general, se parte de una concepción del lenguaje como filtro de la realidad; en palabras de Cook (2004: 2) "the window pane of language, however, is far from being clear and clean. It is often smudged, or curved in ways which distort the images beyond". Este lingüista compara justamente el lenguaje con el cristal de la ventana a través de la que vemos la realidad, no nos centramos en ver el cristal, pero sí vemos a través de él, y de ese mismo modo no nos centramos en las palabras, pero estas pueden distorsionar el mensaje que vehiculan. Además, la asunción de una perspectiva crítica implica asumir que existen ideologías y prácticas dominantes que subordinan y/o excluyen a otras de carácter minoritario, a lo que podríamos denominar "la otredad" en materia de alimentación. La dominación se hace patente en las prácticas hegemónicas como el carnismo, que se asume en general como la normalidad y no como un conjunto de creencias compartidas semejantes a las que subyacen bajo otras prácticas como el vegetarianismo, que se presenta por el contrario como una desviación de la norma. La perspectiva crítica implica asumir, pues, la existencia de modelos hegemónicos, de prácticas alimentarias, modelos físicos y otras formas de vida canónicas que se han naturalizado desde el poder como preeminentes. Esto no resulta óbice para que algunos discursos inicialmente al margen de las ideologías dominantes hayan sabido encontrar su espacio y, en algunos casos, hayan podido imponerse a discursos mayoritarios. En el caso de los discursos de la globalización y la construcción discursiva del capitalismo, es necesario mencionar los trabajos de Fairclough de (2006) y la última edición (2014) de su clásico estudio sobre lengua y poder publicado por primera vez en 1989. De cualquier modo, esta realidad solo supondría la sustitución de unas ideologías por otras, como viene observándose con la tensión entre la defensa y detracción de la alimentación ecológica o de los alimentos genéticamente modificados. Cada ideología se reforzará mediante procesos de argumentación contrapuestos, como así expone Cook (2004) en su investigación sobre los procesos de argumentación en el tratamiento de las tecnologías de cultivo caracterizadas por la realización de modificaciones genéticas de los alimentos. Pero, en definitiva, para la imposición de determinados modelos alimentarios y su posterior difusión es necesario que la ciencia recurra al instrumento del lenguaje, y este ha sido objeto de diferentes estudios que atañen a su dimensión ideológica. 
El lenguaje estructura y condiciona las relaciones sociales y la manera de percibir la realidad, el lenguaje es en definitiva el escultor del pensamiento. Según Butler (2002) el lenguaje provee de un campo de significaciones que, por medio de la reiteración, se convierten en normas que determinan a los sujetos. Esta performatividad del lenguaje responde a una serie de convenciones asumidas con naturalidad por el conjunto de la sociedad que organiza y construye al mundo en polaridades dicotómicas. En el caso de los discursos de la alimentación, esta disposición dicótoma se observa claramente en el tema de la seguridad alimentaria con la polarización positivo/negativo en cuanto a la consideración de los alimentos transgénicos, de los productos ecológicos o de los alimentos funcionales (Muñoz 2002; Henderson \& Johnson 2012); también en el tema de las prácticas alimentarias omnívoras frente a todas las demás, con algunas discusiones basadas en el especismo (Carolina Navarro 2016); o de la homogeneización de la cultura a través de la introducción de prácticas alimentarias hegemónicas enfrentadas a las autóctonas o minoritarias (Durán Monfort 2012). El tema del discurso omnívoro también ha sido discutido por Johnston and Baumann (2007), que observan en la actualidad una tendencia más acorde con hábitos y discursos democráticamente inclusivos frente al actual rechazo de la exclusión, lo cual constituye nuevamente un posicionamiento dicótomo que pone de manifiesto esa tendencia general a organizar la realidad de forma polarizada. En definitiva, esta es una de las vías de investigación que ha sido explorada en cuanto al tema de la alimentación, la contradicción patente en los discursos alimentarios una vez que se analizan en su conjunto y también la contradicción entre la información nutricional excedente y los datos negativos sobre el impacto de la mala alimentación en la salud de las sociedades económicamente desarrolladas, especialmente americana y europea (Gracia Arnaiz 2005).

En la línea de las investigaciones que enfrentan los modelos hegemónicos a los modelos minorizados o propios de la otredad, Mascarenhas (2012) también aborda la cuestión de los discursos de la alimentación en términos de poder en cuanto a la adopción de prácticas alimentarias y plantea la cuestión a partir de la noción de biopolítica de Foucault y, en concreto, desde los procesos de subjetivación que se activan con el discurso de la alimentación. En este sentido, los "biopoderes" (Mascarenhas 2012: 108), que naturalizan aspectos de la vida como la alimentación o la sexualidad, controlarían la sociedad sin necesidad de mecanismos coercitivos o inhibitorios adicionales. Para Foucault (1976), la alimentación se asienta en un discurso de poder identificado con los discursos de las personas expertas en la materia, o lo que es lo mismo, en el discurso científico. Por este motivo es tan relevante la noción de "biopolítica" en muchas de las investigaciones referidas en este artículo, ya que se trata de un concepto que subyace bajo la legitimación y naturalización del discurso especializado en materia de alimentación. En este contexto, el sujeto que recibe los discursos de la alimentación tiende a asumirlos y a producir su propia verdad en un proceso de subjetivación que lleva a normalizar identidades y prácticas. Bourdieu (1991), con la noción de habitus, esquemas interiorizados y naturalizados de la estructura social, definida por la capacidad de producir prácticas sociales en términos, por ejemplo, de gusto, también completa un marco teórico en el que la idea de fondo es la de la interiorización y naturalización de prácticas y modelos de vida, en los que por supuesto nos interesan en concreto los discursos de la alimentación que han servido para potenciar y consolidar modelos culturales o prácticas de consumo. También resulta relevante en las investigaciones lingüísticas e ideológicas de los discursos alimentarios el concepto de "clase social", pues en las prácticas de consumo tam- 
bién entran en juego las identidades sociales y el sentimiento de pertenencia a determinada clase, fundamentales en la definición, por ejemplo, de la clase alta, en la que importan tanto los hábitos y el capital simbólico como el capital económico. En 1946, George Orwell ya apunta la idea del consumo de determinados alimentos, en concreto el té, como parte de una ritualización que conformaba una parte de la identidad personal y cultural. En esta dirección, algunas investigaciones ya se han detenido en analizar cómo las prácticas alimentarias y de consumo estratifican socialmente o forman parte de la construcción identitaria del individuo (Shugart 2015). Como defienden Allen y Anderson (1994: 73), las preferencias de los consumidores y las consumidoras hunden sus raíces en una jerarquía de clase que se impone en la sociedad por lo culturalmente dominante.

En estos contextos descritos, el ser humano es prácticamente heterónomo, es decir, un ser dominado por poderes ajenos que apenas sí puede desarrollarse libremente en términos de prácticas alimentarias. Por un lado, los discursos de la ciencia, bien de alto nivel de especialización bien divulgativos, disponen al individuo no formado en la materia en una posición de inferioridad y, por supuesto, de sumisión frente a la voz experta. Por otro lado, las estructuras de poder cohíben y reprimen las prácticas no hegemónicas, que no están asimiladas por el grueso de la sociedad. Y, finalmente, los hábitos inoculados en la sociedad también inciden en la naturalización y reproducción de prácticas alimentarias. Esta reflexión es central en la filosofía que se halla bajo el tejido de las investigaciones realizadas con perspectiva crítica en el ámbito de los discursos alimentarios. Entre las estrategias discursivas que algunos de estos trabajos han identificado en los discursos alimentarios destacan la maximización de certidumbres y la construcción y proyección del ethos cientifico autorizado (Aguilar y Fregoso 2013; Alcíbar 2004; Cassany 2003; Ciapuscio 1993 y 2001; Cook 2004; Cook, Reed \& Twiner 2009; Estrada 2008; Gallardo 1998; Halliday y Martin 1993; Hyland 1999 y 2002; Mapelli 2004; Muñoz Fagua 2010; Ribeiro de Ávila 2006, entre otros).

Por lo tanto, y entendiendo ideologías como sistemas de creencias socialmente compartidas (van Dijk 2005), resulta un pilar fundamental en el estudio de la ideología en los discursos alimentarios el análisis de los aspectos culturales e ideológicos que se deslizan, de forma consciente o movidos por automatismos, en estos discursos presentes en la vida cotidiana de la mayoría de individuos de las sociedades económicamente desarrolladas. En el ámbito anglosajón, se ha profundizado desde diferentes perspectivas (estudios lingüísticos, pero también sociológicos) en el estudio combinado de aspectos culturales, ideológicos y discursivos en relación con el tema de la alimentación, sobre todo en los trabajos del lingüista Guy Cook publicados entre 2004 y 2010. Como ya se indicó, la mayoría de estos estudios surgen en la primera década del siglo XXI, en la que la globalización es un fenómeno tan imparable que ha supuesto una modificación real en los hábitos de consumo $\mathrm{y}$, en general, en la forma de ver y hablar de la comida. En este sentido, y relacionado con la globalización, alguno de estos estudios se ha detenido en el análisis de la ideología neoliberal que esconden los discursos de política de seguridad alimentaria, como el de Jarosz (2011). En el contexto de Reino Unido, MacMillan y Dowler (2012: 201) concluyen al abordar los discursos de la seguridad alimentaria que el lenguaje y la formulación del discurso sobre políticas de seguridad en la alimentación revelan prioridades y objetivos por parte de los grupos de poder y, en concreto, tras analizar la retórica del discurso gubernamental sobre las políticas de seguridad alimentaria, ponen de manifiesto la audacia del gobierno al hacer 
ver a la población que contribuyen a la formación de un nuevo pensamiento crítico. Por otra parte, la relación entre discursos de la salud y las nociones de género y clase social se abordan en el trabajo de Annandale y Clark (2000). Finalmente, Lupton (2000) realiza el análisis del discurso de la alimentación a partir de la noción de riesgo, un mecanismo habitual de control de la sociedad.

Muchos de los trabajos mencionados recogen ideas relativas a la proyección social de modelos dominantes a través del discurso de la alimentación. Algunos de estos modelos sirvieron, como ya se mencionó, de mecanismo de control y mantenimiento de la clase trabajadora. Otros de los modelos hegemónicos proyectados por los discursos de la ciencia han llevado a cánones físicos y estilos de vida estandarizados que han sido sumamente analizados en diversos trabajos del ámbito de la comunicación y la publicidad (Díaz Rojo y Morant 2007). Como advierten Gracia y Comelles (2007), desde la segunda mitad del siglo XX se ha producido un notable cambio cultural en las representaciones en cuanto a la consideración del cuerpo físico y de sus cuidados, especialmente en lo que se refiere a la mujer, aunque no de forma exclusiva. En el nuevo contexto se presenta un mercado dirigido al cuidado personal más allá de la protección física contra las enfermedades, basado en la permanente exhibición estética y en la preservación de principios éticos y de consumo responsable. Gracia Arnaiz (2007) ya aborda el tema de la construcción social de la normalidad dietética, y aclara uno de los aspectos más polémicos, la promoción de unas prácticas y rutinas de alimentación frente a otras y la naturalización cultural de esas prácticas, que son asumidas como la única opción saludable posible. Se normaliza de este modo la vida cotidiana. Los trabajos sobre la construcción de la alimentación saludable en la publicidad son muy numerosos, y en el ámbito hispanohablante destaca, por ejemplo, la aportación de Díaz Rojo, Morant y Westall (2005). En cuanto a la naturalización ideológica de los discursos alimentarios dominantes, Ferreira da Silva (2013: 250), al tratar la noción de "biopoder" comenta el sometimiento a la visión hegemónica de la ciencia que domina en las sociedades actuales en lo relativo a las normas de alimentación variada, pretendidamente saludable, que tienden además hacia la homogeneización de prácticas.

Esto lleva a una de las grandes temáticas en las investigaciones sobre el discurso de la alimentación, las estrategias lingüísticas que envuelven los géneros discursivos en los que se trata de la alimentación ecológica, también denominada biológica (Nowacek \& Nowacek 2008). La polarización que actualmente se observa en los discursos sobre la alimentación ecológica posee un interés especial en España, ya que se está asistiendo en la divulgación científica al choque -con repercusión mediática- entre críticas feroces al respecto de los beneficios que reporta el consumo ecológico (Mulet 2011) y textos que avalan las bondades de una alimentación con gran presencia de los productos ecológicos. Ya se mencionó que la argumentación discrepante entre defensa y detracción, tanto en lo que respecta al consumo ecológico como, por mencionar otras problemáticas, al carácter saludable e impacto medioambiental de los alimentos transgénicos, por ejemplo, ha sido ampliamente tratada en algunas investigaciones centradas en el ámbito anglosajón como la de Cook (2004). Estos trabajos parten del análisis discursivo para llegar a las lógicas que subyacen en la sociedad en cuanto a la consideración general de la alimentación. Constituyen un puente entre lo lingüístico y lo sociológico, que se complementa con los numerosos estudios de carácter estadístico sobre las prácticas alimentarias y sobre las preocupaciones sobre la salud en las sociedades económicamente desarrolladas. 
Pero, si bien es cierto que en el ámbito anglosajón existe un mayor número de estudios que realizan un abordaje lingüístico y sociológico de esta problemática, en el ámbito hispanohablante no son demasiadas las investigaciones que se han dedicado a ir más allá del dato estadístico para profundizar en los aspectos discursivos de la alimentación. Gracia Arnaiz (2005: 161) afirma en este sentido que "si bien se conocen ciertos datos sobre el consumo de alimentos y el aumento de la incidencia de ciertas enfermedades, se desconoce de una forma absoluta cuáles son las causas que guían las elecciones alimentarias y el modo en que están determinadas por factores socioculturales". Esta autora sostiene que asistimos a una "medicalización del comportamiento alimentario" sostenido por las autoridades a partir del discurso científico. Pero, como la investigadora advierte, las normas de la dieta saludable no existen, la casuística es tal que echa por tierra cualquier intento de generalización (se apoya en las investigaciones realizadas, por ejemplo, sobre el consumo excesivo de sal en Japón, donde un estudio de la OMS de 1986 lo presentó como el país con el índice de enfermedades del corazón más bajo del mundo pese a su elevadísimo consumo de sal). La concepción nutricionista y dogmática de la alimentación y los discursos derivados de esta no tienen en cuenta algunos factores determinantes para la sociedad, como el acceso a los recursos económicos, el entorno laboral, el sexo, la cultura o los hábitos familiares, por lo que estos discursos pueden ser incluso lesivos y facilitar los procesos de estratificación social (Fiallo 1988, Shugart 2015).

Para terminar el panorama general de investigaciones realizadas sobre ideología y discurso alimentario, hay que señalar que en el ámbito hispanohablante son más los trabajos que se han realizado en países americanos que en España. Es el caso de Alvear (2015), que analiza los discursos y prácticas alimentarias en condiciones de pobreza en Santiago de Chile, el estudio de Demonte (2013) sobre la alimentación en el discurso mediático argentino, el trabajo de Théodore et al. (2011) sobre las representaciones sociales a partir de los discursos escolares en México, o los análisis de las representaciones sociales del veganismo y el omnivorismo realizados por Navarro (2013 y 2015), entre otros muy recientes.

\section{A MODO DE CONCLUSIÓN}

Puesto que se trata este trabajo de una revisión de las distintas perspectivas analíticas y dimensiones temáticas de la investigación lingüística en cuanto al ámbito alimentario, no es posible identificar conclusiones al uso, pero sí lo es determinar algunas de las tendencias que se plantean como desafíos de la investigación en el futuro, así como situar el papel de la investigación española en esta materia.

En España, el estudio del discurso alimentario es todavía una línea de investigación reciente. Si bien es cierto que hay multitud de investigaciones realizadas desde una perspectiva antropológica (Benito Morán 2016), desde la comunicación y la publicidad (Díaz Rojo, Morant y Westall 2005 y 2006) o la sociología (Macías 2005), no se observa el mismo desarrollo de la investigación en el ámbito de la lingüística, en el que, a pesar de algunos estudios plenamente lingüísticos como, por ejemplo, el de Cassany y Martí (1998), faltan estudios globales, críticos e interdisciplinares especialmente dirigidos al análisis ideológico del discurso científico y divulgativo de la alimentación y de sus repercusiones sociales. Este es, por tanto, uno de los principales desafíos de la investigación lingüística en el ámbito español. 
En general, en 2019 se observa una clara tendencia de los estudios del discurso de la alimentación en sintonía con la construcción de identidades vulnerables como por ejemplo las de las personas que padecen trastornos alimentarios como la anorexia en entornos comunicativos digitales. En esa línea han trabajado Mullany et ál. (2019) analizando el discurso de jóvenes con anorexia en foros especializados. Por otro lado, la eclosión de la alimentación vegetariana y vegana en ámbitos antes monopolizados por el consumo de carne (pensemos en cadenas como Burger King y sus campañas de hamburguesas vegetales) ha llevado a centrar la atención en cómo los discursos carnistas se vinculaban con pretéritas masculinidades y, lo que es más importante, cómo se está salvando esta barrera desde el marketing a través justamente del uso del lenguaje. Bogueva y Marinova (2019) han analizado desde esta perspectiva el proceso de reconciliación discursiva de la identidad masculina y las prácticas alimentarias más humanitarias, ecologistas y/o veganas. Finalmente, en el contexto español también continúan siendo recurrentes los estudios de la construcción discursiva de la "dieta Mediterránea". Es el caso del estudio de De Moraes Prata Gaspar y Verthein (2019) en el que ponen el foco en las nociones de "salud" y "tradición" en los discursos de dietistas y mujeres de la ciudad de Barcelona.

Como se puede concluir, la presencia del discurso alimentario en redes sociales y medios de comunicación imponen una metodología analítica heterogénea en la que es imprescindible la compilación de corpus textuales de estas procedencias. Cabe señalar en este sentido una última tendencia de investigación en cuanto a discurso alimentario, el estudio de los referidos corpus desde un punto de vista metodológico. Así, Sánchez Sabaté, del Valle y Mensa (2019) centran su aportación en los métodos para localizar y organizar los grandes corpus temáticos de noticias de prensa publicadas sobre el tema de la alimentación. Reducen de este modo el hecho alimentario a una serie de palabras clave que permiten recuperar las noticias a través de buscadores de acceso ordinario y generalizado. De este modo, el análisis de Big Data y el manejo de software de análisis cuantitativo también parecen suponer un nuevo reto para el estudio del discurso alimentario.

Para finalizar, es necesario destacar la necesidad que existe de profundizar en estudios discursivos sobre la alimentación desde una perspectiva crítica, pues es tal la influencia social que poseen los mensajes sobre salud y alimentación que se ha de centrar el interés de las investigaciones lingüísticas en estos aspectos clave para el desarrollo y el bienestar social.

\section{REFERENCIAS BIBLIOGRÁFICAS}

Aguilar, Luz E. y Gilberto Fregoso. (2013). "La lectura de la polifonía e intertextualidad en el texto científico”. Revista Mexicana de Investigación Educativa, vol. 18, núm. 57, pp. 413-435.

Alcaraz Varó, Enrique; José Mateo Martínez y Francisco Yus Ramos (eds.) (2007). Las lenguas profesionales y académicas. Barcelona: Ariel.

Alcíbar, Miguel. (2004). "La divulgación mediática de la ciencia y la tecnología como recontextualización discursiva”. Anàlisi 31, pp. 43-70.

Alcíbar, Miguel. (2007). Comunicar la ciencia. La clonación como debate periodístico. Madrid: Consejo Superior de Investigaciones Científicas.

Alcock, Rupert. (2009). "Speaking Food. A Discourse Analytic Study of Food Security". School of Sociology, Politics, and International Studies, University of Bristol, Working Paper No. 07-09.

Allen, Douglas E. y Paul F. Anderson. (1994). "Consumption and Social Stratification: Bordieu's Distinction". Advances in Consumer Research Volume 21, pp. 70-74. 
Alvear, Katherine. (2015). "Discursos y prácticas alimentarias en hombres y mujeres en condición de pobreza de la Región Metropolitana de Santiago". Revista chilena de nutrición, 42(3), 254-259.

Atienza, Encarnación y T. van Dijk. (2010). "Identidad e ideología en libros de texto españoles de ciencias sociales". Revista de Educación, no 353, pp. 67-106

Benito Morán, Cristina de. (2016). La alimentación como práctica política cotidiana. Análisis antropológico social de los grupos de consumo ecológico. Madrid: Universidad Autónoma de Madrid (Tesis doctoral inédita).

Bazerman, Charles. (1988). Shaping Written Knowledge: The Genre and Activity of the Experimental Article in Science. Wisconsin: The University of Wisconsin Press.

Bloom, Lynn (2008). "Consuming prose: The delectable rhetoric of food writing". College English, 70(4), pp. 346-362.

Bogueva, Diana and Dora Marinova. (2019). "Reconciling Not Eating Meat and Masculinity in the Marketing Discourse for New Food Alternatives". En Bogueva et ál. (2019) Environmental, Health, and Business Opportunities in the New Meat Alternatives Market, IGI Global.

Bourdieu Pierre. (1991). La distinción: Criterios y bases sociales del gusto. Madrid: Taurus.

Brunori, Gianluca; Vanessa Malandrin and Adanella Rossi. (2013). "Trade-off or convergence? The role of food security in the evolution of food discourse in Italy". Journal of Rural Studies 29, pp. 19-29 (Disponible en https://doi.org/10.1016/j.jrurstud.2012.01.013)

Butler Judith. (2002). Cuerpos que importan. Buenos Aires: Paidós.

Carvalho, Anabela. (2007). "Ideological cultures and media discourses on scientific knowledge: rereading news on climate change". Public Understanding of Science, $\mathrm{n}^{\mathrm{o}} 16, \mathrm{pp} .223-243$.

Cassany, Daniel. (2003). "Análisis de la divulgación científica: modelo teórico y estrategias divulgativas". En Texto, Lingüistica y cultura. XIV Congreso de la Sociedad Chilena de Lingüística. Comunicaciones seleccionadas. Osorno: Editorial Universidad de Los Lagos, pp. 57-80.

Cassany, Daniel y Jaume Martí. (1998). "Estrategias divulgativas del concepto prión". En Actas del I Congreso Internacional de Análisis del Discurso, Madrid: Universidad Complutense, pp. 58-66.

Chamorro, Diana; Norma Barletta y Jorge Mizuno. (2013). "El lenguaje para enseñar y aprender las Ciencias Naturales: Un caso de oportunidades perdidas para la formación ciudadana". Revista Signos. Estudios de Lingüística 46 (81), pp. 3-28.

Ciapuscio, Guiomar E. (1993). "Reformulación textual: El caso de las noticias de divulgación científica". Revista Argentina de Lingüística, 9 (1-2): 69-116.

Ciapuscio, Guiomar E. (2001). "Procesos y Recursos de Producción Textual en la Divulgación de Ciencia”. En J. Brumme (ed.), La historia de los lenguajes iberorománicos de especialidad. La divulgación de ciencia, Barcelona: Vervuert/Iberoamericana, pp. 17-42.

Cook, Guy. (2004). Genetically modified language. The discourse of arguments for GM crops and food. London and New York: Routledge.

Cook, Guy. (2007). "Organic shoppers want facts, not just stories”. Organic and Natural Business 2007, pp. 6-7.

Cook, Guy. (2010). "Sweet talking: Food, language, and democracy”. Language Teaching, 43:2, pp. 168-181.

Cook, Guy; Elisa Pieri and Peter T. Robbins. (2004). “The scientists think and the public feels': expert perceptions of the discourse of GM food". Discourse \& Society, vol. 15, 4, pp. 433-449.

Cook, Guy; Peter T. Robbins and Elisa Pieri. (2006). "Words of mass destruction": British newspaper coverage of the genetically modified food debate, expert and non-expert reactions". Public Understanding of Science, vol. 15, 1, pp. 5-29.

Cook, Guy; Matt Reed and Alison Twiner. (2009). "But it's all true!": commercialism and commitment in the discourse of organic food promotion". Text \& Talk. An Interdisciplinary Journal of Language, 29 (2), pp. 151-173. 
De Choudhury, Munmun; Sanket Sharma and Emre Kiciman. (2016). "Characterizing Dietary Choices, Nutrition, and Language in Food Deserts via Social Media”. CSCW '16, 2016, San Francisco, pp. 1157-1170.

De Moares Prata Gaspar, María Clara y Úrsula Verthein. (2019). "Entre la "salud" y la "tradición": las representaciones sociales de la dieta mediterránea". Physis 29 (02) doi.org/10.1590/S010373312019290217

Demonte, Flavia. (2013). "La alimentación como problema en el espacio mediático: análisis de editoriales de la prensa gráfica argentina, 2002-2008". Signo y Pensamiento 62, pp. 82-97.

Díaz Ramírez, Glenda.; María C. Souto-Gallardo; Montserrat Bacardí Gascón; y Arturo Jiménez-Cruz. (2011). "Efecto de la publicidad de alimentos anunciados en la televisión sobre la preferencia y el consumo de alimentos: revisión sistemática". Nutrición hospitalaria 26 (6), pp. 1250-1255.

Díaz Rojo, J. Antonio y Ricard Morant. (2007). "El discurso crítico contra la 'tiranía' del culto al cuerpo". Tonos Digital 14, s/p (disponible en http://www.um.es/tonosdigital/znum14/secciones/ estudios-8-cuerpo.htm).

Díaz Rojo, J. Antonio, Ricard Morant y Debra Westall. (2006). "You are what you eat! Health Claims and Spanish Nutritional Discourse". En J. L. Blas Arroyo, Manuela Casanova Ávalos y Mónica Velando Casanova (eds.) Discurso y sociedad. Contribuciones al estudio de la lengua en contexto social. Castelló: Universitat Jaume I.

Díaz Rojo, J. Antonio, Ricard Morant y Debra Westall. (2005). "El sanismo' lingüístico: recursos retóricos en la publicidad y etiquetado de los alimentos". Revista de investigación lingüística VIII, pp. 35-51. (Disponible en http://revistas.um.es/ril/article/viewFile/6661/6461).

Durán Monfort, Paula. (2012). Representaciones, discursos y saberes en torno a la alimentación en Túnez: Modelo hegemónico y conocimientos ausentes. Tarragona: Universitat Rovira i Virgili.

Eberenz, Rolf. (2014) "Alimentos, platos y bebidas en el Tesoro de la lengua castellana o española (1611) de Sebastián de Covarrubias". En Rolf Eberenz (ed.) Discursos hispánicos sobre alimentación y culinaria. Aproximaciones literarias y lingüisticas. Madrid: Visor Libros, pp. 385-407.

Estrada, Andrea. (2008). "Ethos y discurso científico-académico. Los marcadores de evidencialidad como estrategia de cortesía". Revista de la Sociedad Argentina de Lingüística $\mathrm{N}^{\mathrm{o}} 1 / 2-2008$, pp. 7-24.

Fairclough, Norman. (1995). Critical Discourse Analysis. London: Longman.

Fairclough, Norman. (2006). Language and globalization. London: Routledge.

Fairclough, Norman. (2014, $3^{\mathrm{a}}$ ed.). Language and Power. London: Longman.

Febrer Fernández, Nieves. (2014). "El estudio de la alimentación a través del análisis de textos fílmicos". Historia y Comunicación Social vol. 19, pp. 181-193.

Ferreira da Silva, Luisa (2013). "Food and culture: change and continuity". Demetra 2013; 8(Supl.1), pp. 247-255.

Fiallo, Ana Teresa. (1988). "Linguistics, Language, and Discrimination. Ideology and the Social Effects of Linguistic Scientific Discourse”. Equity \& Excellence in Education Vol. 24 (4), pp. 5-7.

Foucault, Michel (1976) Histoire de la sexualité, 1, La Volonté de savoir. Paris: Gallimard.

Frye, Joshua and Michael Bruner (ed.). (2012). The Rhetoric of Food. Discourse, Materiality, and Power. New York and London: Routledge.

Gallardo, Susana (1998). "Estrategias y procedimientos de reformulación en textos de divulgación científica". Revista de la Sociedad Argentina de Lingüística,1, pp. 67-79.

García Negroni, María Marta. (2008). "Subjetividad y discurso científico-académico. Acerca de algunas manifestaciones de la subjetividad en el artículo de investigación en español". Revista Signos 41(66), pp. 5-31.

García Negroni, María Marta. (2009). "Reformulación parafrástica y no parafrástica y ethos discursivo en la escritura académica en español. Contrastes entre escritura experta y escritura universitaria avanzada". Letras de Hoje, v. 44 (1), pp. 46-56. 
Gómez Oroná, Iñaki J. (2014). "Lo alimentario, la construcción de una dimensión humana en el discurso publicitario contemporáneo". En XVIII Jornadas Nacionales de Investigadores en Comunicación. Área Transdepartamental de Crítica de Artes (IUNA), s/p.

González Ramírez, Carolina. (2013). "Procedimientos de reformulación en textos de divulgación didáctica". Tejuelo 17, pp. 9-24.

Gouveia, Carlos A. M. y Marta F. Alexandre (2009). "The Arrogant Scientist and the Ignorant Citizen: A Critical Discourse Analysis of the Discourse of Scientists". En Alcinda Pinheiro de Sousa et al., So long lives this, and this gives life to thee: Homenagem a Maria Helena de Paiva Correia. Lisboa: Edições Colibri, pp. 177-190.

Gouveia, Carlos A. M. (2003). "Critical Discourse Analysis and the Development of the New Science". En Weiss, Gilbert \& Ruth Wodak (eds.) Critical Discourse Analysis: Theory and Interdisciplinarity. London: Palgrave Macmillan, pp. 47-62.

Gracia Arnaiz, Mabel. (2005). "Maneras de comer hoy. Comprender la modernidad alimentaria desde y más allá de las normas”. Revista Internacional de Sociología, 40, pp. 159-182.

Gracia Arnaiz, Mabel. (2007). "Comer bien, comer mal: la medicalización del comportamiento alimentario". Salud pública de México vol.49, no.3, mayo-junio de 2007, pp. 236-242.

Gracia Arnaiz, Mabel y Josep M. Comelles (eds.) (2007). No comerás. Narrativas sobre comida, cuerpo y género en el nuevo milenio. Barcelona: Icaria Editorial y Universitat Rovira i Virgili.

Jacobi, Daniel. (1987). Textes et images de la vulgarisation scientifique. Berna: Peter Lang.

Jarosz, Lucy. (2011). "Defining World Hunger Scale and Neoliberal Ideology in International Food Security Policy Discourse". Food, Culture and Society Volume 14 (1), pp. 117-139.

Johnston, Josée and Shyon Baumann, "Democracy versus Distinction: A Study of Omnivorousness in Gourmet Food Writing". American Journal of Sociology 113, no. 1 (July 2007), pp. 165-204.

Luke, Allan. (2002). "Beyond science and ideology critique: developments in Critical Discourse Analysis". Annual Review of Applied Linguistics 22, pp. 96-110.

Hahn, L. K. and Bruner, M. (2012). "Politics on Your Plate: Building and Burning Bridges across Organic, Vegetarian, and Vegan Discourse". En Joshua J. Frye and Michael S. Bruner (eds.) The Rhetoric of Food. Discourse, Materiality, and Power. New York, London: Routledge, pp. 42-58.

Halliday, Michael A. K. (1985). An introduction to functional grammar. London: Edward Arnold.

Halliday, Michael A. K. and Jim R. Martin. (1993). Writing science: Literacy and discursive power. London: Falmer.

Henderson, A. and Vanessa Johnson. (2012). "Food, Health, and Well-Being. Positioning Functional Foods". En Joshua J. Frye and Michael S. Bruner (eds.) The Rhetoric of Food. Discourse, Materiality, and Power. New York, London: Routledge, pp. 71-89.

Hyland, Ken. (2002). "Authority and invisibility: authorial identity in academic writing". Journal of Pragmatics 34, pp. 1091-1112.

Hyland, Ken. (1999). "Academic Attribution: Citation and the Construction of Disciplinary Knowledge". Applied Linguistics 20/3, pp. 341-367).

Leigh Burton, Katelyn. (2016). You Are What You Eat: Investigating Food Discourse and DigitallyMediated Identities. Open Access Dissertation. University of Rhode Island.

Lupton, Deborah. (2000). "Food, risk, and subjectivity". En Williams, Simon J.; Jonathan Gabe and Michael Calnan (ed.) Health, medicine and society, key theories, futur agendas. Londres: Routledge, pp. 205-218.

Luz, Madel T; Alcindo A. Ferla; Anderson dos Santos Machado and Rafael Dall Alba. (2017). "Retórica na divulgação científica do imaginário de vida e saúde: uma proposta metodológica de análise". Interface 21(61), pp. 333-47.

Macías, Olga. (2005). "Alimentación e ideología obrera en Bilbao durante la transición del siglo XIX al XX”. Zainak 27, pp. 251-268. 
MacMillan, Tom and Elizabeth Dowler. (2012). "Just and Sustainable? Examining the Rhetoric and Potential Realities of UK Food Security". Journal of Agricultural and Environmental Ethics 25, pp. 181-204.

Mapelli, Giovanna. (2004). "Estrategias lingüístico-discursivas de la divulgación científica”. Actas del XXII Congreso AISPI, pp. 169-184.

Marinkovich, Juana. (2005). "Las estrategias de reformulación: el paso desde un texto-fuente a un texto de divulgación didáctica”. Literatura y Lingüítica n 16, pp. 191-210.

Martín Criado, Enrique. (2007). "El conocimiento nutricional apenas altera las prácticas de alimentación: el caso de las madres de clases populares de Andalucía". Revista Española de Salud Pública $\mathrm{n}^{\mathrm{o}}$ 5, pp. 519-528.

Martín Criado, Enrique. (2010). "Las tallas grandes perjudican seriamente la salud. La frágil legitimidad de las prácticas de adelgazamiento entre las madres de clases populares". Revista Internacional de Sociología Vol. 68 (2), pp. 349-373.

Mascarenhas, Maria Paula de Vilhena. (2012). "Alimentación y dietética en los procesos de subjetivación”. En Mabel Gracia Arnaiz (ed.) Alimentación, salud y cultura: encuentro interdisciplinares, Colección Antropologia Mèdica, 7, Tarragona, pp. 103-119.

Mazzacano D'Amato, Pierre \& Joseph Falzon. (2015). "Why do some consumers prefer organic food? A Discourse Analytical Perspective". Journal of Food Products Marketing 21, issue 3, pp. 255-273.

Moss, Gillian. (2010). "Textbook language, ideology and citizenship: the case of a history textbook in Colombia”. Functions of Language 17 (1), pp. 71-93.

Moss, Gillian; Norma Barletta; Diana Chamorro; y Jorge Mizuno. (2013). "La metáfora gramatical en los textos escolares de Ciencias Sociales en español”. Onomázein 28, pp. 88-104.

Moya de Sifontes, Mary Zulay. (1986). "Efecto de los medios de comunicación social en la adquisición de alimentos a nivel familiar". Archivos latinoamericanos de nutrición, 36 (I), pp. 166-186.

Mulet, José Miguel. (2011). Los productos naturales jvaya timo!, Pamplona: Laetoli.

Mullany, Louise et ál. (2019). “'Am I anorexic?’: weight, eating and discourses of the body in online adolescent health communication". Communication and Medicine, 12 (2-3) doi:10.1558/ cam. 16692

Muñoz, Carlos. (2002). "El debate ideológico de la seguridad alimentaria". Puntoycoma 77, s/p [último acceso 11/04/2017, disponible en http://ec.europa.eu/translation/bulletins/puntoycoma/77/ pyc776_es.htm].

Muñoz Dagua, Clarena. (2010). "El rol de la metáfora léxica en la divulgación de la ciencia". Tabula Rasa 13, pp. 273-292.

Navarro, Alexandra X. C. (2013). "Nuevas arenas de disputa por el sentido: discursos veganos y omnívoros en relación al habitus alimentario". Revista Latinoamericana de Estudios Críticos Animales 1: 1-12.

Navarro, Alexandra X. C. (2016). "Carnismo y educación especista: redes de significaciones en las representaciones sociales que estructuran el especismo antropocéntrico en Argentina". Revista Latinoamericana de Estudios Críticos Animales, Año II, Volumen II: mayo 2016, s/p.

Navas López, Julia. (2010). "El experto alimentario y los medios de comunicación". Athenea Digital (18), pp. 49-64.

Nowacek, David, and Rebecca Nowacek (2008). "The organic foods system: Its discursive achievements and prospects". College English 70(4), pp. 403-420.

Orwell, George. (1946). "A nice cup of tea”. Evening Standard (12 enero 1946).

Oseguera Parra, David; (2001). “La comida: ¿lugar de encuentro entre disciplinas científicas?”. Estudios sobre las Culturas Contemporáneas, pp. 141-151.

Pérez Martínez, Alina. (2011). "La comunicación sobre la salud en medios digitales cubanos". Revista Cubana de Salud Pública.

Ribeiro de Ávila Veloso, Simone. (2006). O ethos en entrevistas publicadas em Veja: a divulgação da ciêcia no campo jornalístico. São Paulo: Universidade de São Paulo. 
Rivarosa Alcira, Susana y Ana Lía De Longhi. (2006). “La noción de alimentación y su representación en alumnos escolarizados”. Revista electrónica de enseñanza de las ciencias Vol. 5 (3), pp. 534552.

Roslyng, Mette Marie. (2011). "Challenging the Hegemonic Food Discourse: The British Media Debate on Risk and Salmonella in Eggs". Science as Culture 20, 2, pp. 157-182.

Rousseau, Signe (2012). Food and Social Media: You are What You Tweet. Lanham, MD: AltaMira Press.

Sánchez Sabaté, Rubén, Carlos del Valle y marta Mensa. (2019). "Método para la construcción de grandes corpus temáticos de noticias de prensa digital. Hacia un corpus sobre el hecho alimentario". Revista Latina de Comunicación Social 74, pp 594-617.

Schaefer, Somia C. (2012). Historia de la educación alimentaria. El discurso médico en torno a la alimentación del niño entre 1875 y 1930 en Argentina.Tesis de Licenciatura. Universidad de San Andrés.

Scollon, Ron. (2005). "The discourses of food in the world system. Toward a nexus analysis of a world problem". Journal of Language and Politics 4 (3), pp. 465-488.

Shugart, Helene A. (2015). "Food Fixations. Reconfiguring Class in Contemporary US Food Discourse". Food, Culture \& Society 17,2, pp. 261-281.

Szatrowski, Polly E. (ed.) (2014). Language and Food: Verbal and nonverbal experiences. Amsterdam and Philadelphia: John Benjamins.

Théodore, Florence L; Anabelle Bonvecchio Arenas; Ilian Blanco García; Yohani Carreto Rivera. (2011). "Representaciones sociales relacionadas con la alimentación escolar: el caso de las escuelas públicas de la Ciudad de México". Salud colectiva, vol. 7 (2), pp. 215-229.

van Dijk, Teun A. (2003). Ideología y discurso. Barcelona: Ariel.

van Dijk, Teun A. (2005). "Ideología y análisis del discurso". Revista Internacional de Filosofía Iberoamericana y Teoría Social $\mathrm{n}^{\circ}$ 29, pp. 9-36.

Williams, Simon J.; Jonathan Gabe and Michael Calnan (ed.) (2000). Health, medicine and society, key theories, futur agendas. Londres: Routledge. 
